\section{MORBIMORTALIDADE HOSPITALAR ENTRE CRIANÇAS E ADOLESCENTES POR TRAUMATISMO INTRACRANIANO NO ESTADO DA BAHIA, BRASIL}

\section{HOSPITAL MORBIMORTALITY IN CHILDREN AND ADOLESCENTS BY INTRACRANIAL TRAUMATISM IN THE STATE OF BAHIA, BRAZIL}

Aline Gomes Santos ${ }^{1}$ / Ana Paula Santos Coelho ${ }^{1}$ / Glasiele Santos de Oliveira ${ }^{1} /$ Mariana da Silva Santos ${ }^{1 /}$ Ricardo Bruno Santos Ferreira ${ }^{1, *} /$ Rubia Pinto Carvalho ${ }^{1}$

\section{INTRODUÇÃO}

O conceito de traumatismo cranioencefálico (TCE) refere-se a qualquer acometimento traumático que ocasione lesão física, com comprometimento das funções cerebrovasculares de um indivíduo (NETO et al., 2016).

Os traumas são decorrentes de lesões fechadas ou penetrantes e tais lesões cranianas e encefálicas podem implicar em dano neural, insuficiência vascular e efeitos inflamatórios. Dentre as lesões cranianas mais comuns, é possível citar a concussão, contusão, fraturas de crânio, hematoma epidural ou subdural, hemorragia subaracnoide e herniação (MELO, 2006).

É classificado como um problema de saúde pública com grande magnitude e transcendência, com importante impacto na morbidade e mortalidade da população (HORA; SOUSA, 2012). Dentre os grupos acometidos, as crianças e os adolescentes são o segundo grupo etário mais atingido por este agravo no Brasil (MELO, 2014).

Diante do processo de desenvolvimento econômico, com as modificações nos meios de transporte e as novas tecnologias, houve um aumento do número de vítimas de traumas mecânicos; além disso, aumentou-se o quantitativo de mortes violentas, que estão entre as principais causa de óbitos e sequelas na população, repercutindo no nível socioeconômico das vítimas (SANTOS et al., 2013). Outras causas comuns que levam ao traumatismo, são as agressões físicas, violência urbana e quedas em crianças e adolescentes (MELO, 2014).

\begin{abstract}
RESUMO
Objetivo: Descrever a morbimortalidade hospitalar de crianças e adolescentes entre os anos de 2014 a 2018 no estado da Bahia por traumatismo cranioencefálico. Materiais e métodos: Trata-se de um estudo epidemiológico realizado por meio de consulta à dados secundários, junto ao sistema de informação hospitalar (SIH-SUS), disponibilizado pelo DATASUS. Foram consultados os dados referentes ao período de 2014 a 2018. Os dados obtidos foram reorganizados no Microsoft Office Excel e analisados por meio de estatística descritiva. Resultados: Dentro do período analisado, foram registrados no SIH-SUS, 7.879 casos de internações e 316 de óbitos por traumatismo intracraniano em menores de 19 anos. Ocorreu um decréscimo, dentre o período estudado, nas internações e óbitos. A macrorregião leste foi a que obteve os maiores registros de internações $(n=2.034$; $25,8 \%)$, óbitos ( $\mathrm{n}=61 ; 19,3 \%)$, quanto ao caráter de atendimento urgência apresentou $(n=7.481 ; 94,9 \%)$ e óbitos $(n=300 ; 94,9 \%)$. Conclusão: Faz necessário ações governamentais para estimular a prevenção desse agravo e reduzir a subnotificação presente em alguns locais.
\end{abstract}

Palavras-chave: Traumatismos Craniocerebrais. Criança. Adolescente

\section{ABSTRACT}

Objective: To describe the hospital morbidity and mortality of children and adolescents between 2014 and 2018 in the state of Bahia due to traumatic brain injury. Materials and methods: This is an epidemiological study carried out by consulting secondary data, together with the hospital information system (SIH-SUS), made available by DATASUS. The data referring to the period from 2014 to 2018 were consulted. The data obtained were reorganized in Microsoft Office Excel and analyzed using descriptive statistics. Results: Within the analyzed period, 7,879 cases of hospitalizations and 316 deaths from intracranial trauma in children under 19 years were registered in SIH-SUS. There was a decrease, among the studied period, in hospitalizations and deaths. The eastern macro-region was the one with the highest records of hospitalizations ( $\mathrm{n}=2,034 ; 25.8 \%)$, deaths ( $\mathrm{n}$ $=61 ; 19.3 \%)$, regarding the character of urgent care presented $(\mathrm{n}=7,481 ; 94.9 \%)$ and deaths $(\mathrm{n}=300 ; 94.9 \%)$ Conclusion: It is necessary government actions to stimulate the prevention of this disease and reduce the underreporting present in some places.

Keywords: Craniocerebral Trauma. Child. Adolescent.

Submetido em: 08 de nov. 2019

Aceito em: 14 de fev. 2020

${ }^{1}$ Universidade do Estado da Bahia - UNEB, Guanambi, Bahia - Brasil.

*E-mail para correspondência: ricardobrunoenf@gmail.com 
Ainda existem poucos estudos que abordem essa temática no Brasil, apesar da elevação dos números de traumatismos intracranianos em crianças e adolescentes (MELO, 2014; MACHADO FILHO, 2010; MELO, 2006). Neste sentido, conhecer o perfil das vítimas de traumatismos intracranianos e suas principais causas possui relevância na implementação de estratégias para diagnóstico, tratamento, e ações de controle e prevenção. Além disso, servirão de subsídios na atuação dos profissionais de saúde para que estes busquem a redução deste agravo na sociedade (SANTOSet al., 2013), principalmente entre a população jovem.

Diante disso, o presente estudo objetiva descrever a morbimortalidade hospitalar de crianças e adolescentes entre os anos de 2014 a 2018 no estado da Bahia.

\section{MATERIAIS E MÉTODOS}

Este é um estudo epidemiológico descritivo com dados secundários, obtidos por meio de consulta ao SIHSUS (Sistema de Informações Hospitalares do Sistema Único de Saúde) e disponibilizados pelo Departamento de Informática do SUS (DATASUS). A consulta às informações no DATASUS foi realizada nos meses setembro a outubro de 2019.

Foram selecionados os dados por local de residência concernentes as hospitalizações no estado da Bahia devido aos CID-10: traumatismo intracraniano, ocorridas em menores de 19 anos no período de 2014 a 2018. Os dados coletados no SIH-SUS foram sistematizados em planilha do Microsoft Office Excel, o que subsidiou os cálculos de frequências absolutas e relativas.
Foram estudadas as variáveis concernentes ao ano de atendimento, faixa etária, sexo, raça/cor, macrorregião de saúde e caráter de atendimento. Por se tratar de dados secundários e de domínio público, sem identificação dos indivíduos, não foi necessário submeter o estudo ao Comitê de Ética em Pesquisa.

\section{RESULTADOS E DISCUSSÃO}

Foram registrados no período de 2014 a 2018, 7.879 internações hospitalares e 316 óbitos por traumatismos intracranianos entre menores de 19 anos no estado da Bahia, o que corresponde a $6,6 \%$ para as internações e $7,1 \%$ dos óbitos quando analisamos a nível Brasil.

Conforme visualizado na tabela 1, ocorreu uma queda significativa dos registros de internações por TCE em crianças e adolescentes, tanto para os óbitos, com redução de $24,4 \%$ (n=77) para $13,9 \%(\mathrm{n}=44)$, quanto para as internações, com variação de $23,9 \%$ $(n=1.883)$ para $15,3 \%(n=1.205) d u-$ rante o período estudado. Através da análise anual, foi possível identificar que em 2014 ocorreram mais internações $(n=1.883 ; 23,9 \%)$ por traumatismo intracraniano. No que se referem aos óbitos, os anos de 2014 e 2015 houve prevalência de 24,4\% ( $n=77)$, por este agravo.

Acredita-se que tal redução pode ser explicada a partir do estudo de Winn et al (2011), quando pontuaram que ocorreram melhorias nas ações de prevenção, diagnóstico e tratamento às vítimas de TCE, principalmente quanto aos atendimentos nas emergências; apesar de ainda se manter elevado o surgimento do número de novos casos deste agravo.
Tabela 1 - Internações hospitalares por traumatismo intracraniano por ano em menores de 19 anos entre o período de 2014 a 2018 na Bahia.

\begin{tabular}{lc|cc|c} 
& \multicolumn{2}{c}{ Internações } & \multicolumn{1}{c}{ Óbitos } \\
\hline \multirow{2}{*}{ Ano atendimento } & $\mathbf{N}$ & $\mathbf{\%}$ & $\mathbf{n}$ & $\mathbf{\%}$ \\
\cline { 2 - 5 } 2014 & 1.883 & 23,9 & 77 & 24,4 \\
2015 & 1.750 & 22,2 & 77 & 24,4 \\
2016 & 1.602 & 20,3 & 68 & 21,5 \\
2017 & 1.439 & 18,3 & 50 & 15,8 \\
2018 & 1.205 & 15,3 & 44 & 13,9 \\
\hline \multirow{2}{*}{ Total } & $\mathbf{7 . 8 7 9}$ & $\mathbf{1 0 0 , 0}$ & $\mathbf{3 1 6}$ & $\mathbf{1 0 0 , 0}$ \\
\hline \multicolumn{2}{l}{ Fonte: Ministério da Saúde - Sistema de Informa- } \\
ções Hospitalares do SUS (SIH/SUS), 2019.
\end{tabular}

Diante da análise das características sociodemográficas (tabela 2), foi possível identificar que os maiores registros para as internações e óbitos por TCE, foram em crianças e adolescentesdo sexo masculino $(n=5607$; $71,2 \%$ ) e com faixa etária entre 15 a 19 anos $(\mathrm{n}=2.986 ; 37,95 \%)$. Porém, houve o predomínio da subnotificação quanto àraça/cor para internações e óbitos, correspondendo a $53,1 \%$ das internações e 66,5\% dos óbitos, seguido da raça/cor parda com $(n=3.175 ; 40,3 \%)$ das internações e $(n=86 ; 27,2 \%)$ dos óbitos.

Corroborando com o que foi encontrado na presente investigação sobre o predomínio do sexo masculino na ocorrência do TCE, um estudo realizado sobre as internações por traumatismo cranioencefálico em Sergipe identificou que os homens se envolvem mais facilmente em situações violentas e agressivas que podem influenciar em acidentes. Ademais, os homens desde crianças são independentes e tem mais contato com causas externas incluindo o fato de começar a trabalhar cedo e passar ter comportamentos de risco como dirigir alcoolizado (VIANA; BOHLAND, PEREIRA, 2014). 
Tabela 2 - Características sociodemográficas da morbimortalidade hospitalar por traumatismo intracraniano por sexo, faixa etária e raça/cor em menores de 19 anos entre o período de 2014 a 2018 na Bahia.

\begin{tabular}{cc|cc|c} 
& \multicolumn{2}{c}{ Internações } & \multicolumn{2}{c}{ Óbitos } \\
\hline Sexo & $\mathbf{n}$ & $\mathbf{\%}$ & $\mathbf{n}$ & $\%$ \\
\cline { 2 - 5 } Masculino & 5.607 & 71,2 & 250 & 79,1 \\
Feminino & 2.272 & 28,8 & 66 & 20,9 \\
Faixa etária & & & & \\
Menor 1 ano & 510 & 6,5 & 12 & 3,8 \\
1 a 4 anos & 1.634 & 20,7 & 26 & 8,2 \\
5 a 9 anos & 1.415 & 18,0 & 18 & 5,7 \\
10 a 14 anos & 1.334 & 16,9 & 31 & 9,8 \\
15 a 19 anos & 2.986 & 37,9 & 229 & 72,5 \\
Cor/raça & & & & \\
Branca & 324 & 4,1 & 11 & 3,5 \\
Preta & 86 & 1,1 & 6 & 1,9 \\
Parda & 3.175 & 40,3 & 86 & 27,2 \\
Amarela & 110 & 1,4 & 3 & 0,9 \\
Sem informação & 4.184 & 53,1 & 210 & 66,5 \\
\hline Fonte: Ministerio
\end{tabular}

Fonte: Ministério da Saúde - Sistema de Informações Hospitalares do SUS (SIH/SUS), 2019.

Com relação a faixa etária, estudo cearense, identificou que os adolescentes de 15 a 19 anos, tiveram lesões mais graves de TCE, devido estarem mais envolvidos em acidentes de trânsito com motocicletas e sem uso de proteção; também foi possível observar a participação em atos de violência física, decorrentes da falta de serviços de suporte a este público (MACHADO FILHO et al., 2010).

$\mathrm{O}$ alto percentual de subnotificação se assemelha aos achados de outro estudo, que foi observado um alto índice de subnotificação na região Nordeste, devido o fato da região apresentar altos índices de declarações de óbitos por causas indefinidas. Além disso, ocorre também o preenchimento incompleto dos sistemas de notificações, o que acabam alterando o perfil epidemiológico da região e geram falsas informações que afetam diretamente os sistemas de notificações (VIANA; BOHLAND, PEREIRA, 2014).

Passando a se tratar da região de saúde, a macrorregião leste que compreende ao núcleo regional de saúde de Salvador, obteve os maiores registros de internações $(n=2.034 ; 25,8 \%)$ e óbitos ( $n=61 ; 19,3 \%)$. A macrorregião que apresentou menores registro de internações $(n=250 ; 3,2 \%)$ e com maiores taxas de mortalidade $(7,2 \%)$ foi a macrorregião Nordeste que compreende o núcleo regional de saúde de Alagoinhas

Isso se explica pelo fato da região ser grande centro de referência na assistência à saúde, sendo base para os cuidados especializados para diversas situações, incluindo o trauma cranioencefálico, devido ao suporte técnico, profissional e estrutural ser maior nessa região. Todavia, outros fatores também têm contribuído para o índice elevado de TCE nessa região, como acidentes de trânsito, agressões físicas e quedas da própria altura (MELO; SILVA; MOREIRA, 2004).

Quanto ao caráter de atendimento, os maiores registros de internações hospitalares e óbitos, foram em urgência com 7.481(94,9\%) e $300(94,9 \%)$, respectivamente. Por ser a porta de entrega das unidades de saúde, os serviços de urgências são considerados umas das áreas mais amplas e complexas no contexto hospitalar, há uma maior atividade de profissionais de diferentes especialidades, sendo a assistência prestada de forma imediata e eficaz, por meio dela são utilizados recursos tecnológicos e estruturais que contribuem para um bom atendimento (MOLL, 2015).

\section{CONCLUSÃO}

O traumatismo intracraniano em crianças e adolescentes foi causa de mais internações no ano de 2014, no que se referem aos óbitos, os anos de 2014 e 2015 figuraram com uma maior prevalência. No que se refere às características sociodemográficas, os maiores registros para internações e óbitos por TCE foram predominantes em crianças e adolescentes do sexo masculino, faixa etária entre 15 a 19 anos e a raça/cor teve predomínio da subnotificação seguida da raça/cor negra. A macrorregião leste obteve os maiores registros de internação e óbitos já a nordeste apresentou os menores registros de internação e óbitos. Quanto ao caráter de atendimento, os maiores registros de internações hospitalares e óbitos foram em urgência.

Através desses achados podemos perceber que houve um decréscimo na ocorrência do TCE em crianças e adolescentes com o passar dos anos, mas ainda se faz necessário ações governamentais para estimular a prevenção desse agravo, uma vez que a subnotificação se faz presente em alguns locais e acaba gerando falsos resultados. Campanhas devem ser idealizadas para alertar a população no geral, para diminuir ainda mais a ocorrência das internações e óbitos pelo TCE, causa geradora de problemas permanentes que podem ser evitados.

\section{REFERÊNCIAS}

HORA, E.C.; SOUSA, R.M.C. Necessidades das famílias após o Trauma Cranioencefálico: dados da realidade Brasileira. Enferm Foco, v. 2, n.3, p.88-92, 2012.

MACHADO FILHO, J.Á. et al. Perfil clínico-epidemiológico das crianças e adolescentes hospitalizados por traumatismo crânio encefálico. RBPS, v.23, n.4, p. 335-342, 2010.

MELO, J.R.T. Traumatismo craniano na infância. Revista Brasileira de Neurologia e Psiquiatria, v. 18, n.2, p.122-129, 2014.

MELO, J.R.T.; SILVA, R.A.S.; MOREIRA, E.D. Características dos pacientes com trauma cranioencefálico na cidade do Salvador, Bahia, Brasil. 
Arq.Neuropsiquiatria, v. 62, n. 3-A, p.711-715, 2004.

MELO, J.R.T.et al. Traumatismo cranioencefálico em crianças e adolescentes na cidade do Salvador - BAHIA.

ArqNeuropsiquiatr, v. 64, p. 994-6, 2006.

MOLL, A.V.S. Perfil do atendimento dos pacientes com traumatismo cranioencefálico nos hospitais de urgência e emergência sob a gestão da Secretaria de Estado de Saúde do Rio de Janeiro. 78 f. Dissertação apresentada com vistas à obtenção do título de Mestre Modalidade Profissional em Saúde Pública. Fundação Oswaldo Cruz. Rio de Janeiro, 2015.

NETO, C. D. M. et al. Epidemiologia do traumatismo cranioencefálico no Brasil. In: I CONGRESSO NACIONAL DE ESPECIALIDADES EM FISIOTERAPIA, 1., 2016, João Pessoa. Anais... João Pessoa, 2016, p. 386- 403.

SANTOS, F.et al. Traumatismo cranioencéfalico: causas e perfil das vitimas atendidas no pronto-socorro de Pelotas/ Rio Grande do Sul, Brasil. Revista Mineira de enfermagem; 2013.

VIANA, N.J.; BOHLAND, A.K.; PEREIRA, C.U. Internações por traumatismo cranioencefálico em Sergipe, de 2000 a 2011. Arq.Bras. Neurocir, v. 33, n.4, p. 306-317, 2014.

WINN, H.R. et al. Youmans Neurological Surgery: Chapter 323 - Epidemiology of Traumatic Brain Injury". Elsevier Saunders, v. 4, p. 32703275, 2011. 